\title{
Translational Regulation of XIAP Expression and Cell Survival During Hypoxia in Human Cholangiocarcinoma
}

\author{
CARLA MARIENFELD,* YOKO YAMAGIWA,* YOSHIYUKI UENO, ${ }^{*}$ VALORIE CHIASSON,* \\ LINDA BROOKS,* FANYIN MENG,* and TUSHAR PATEL* \\ *Scott and White Clinic, Texas A\&M University System Health Science Center College of Medicine, Temple, Texas; and ${ }^{\star}$ Division of \\ Gastroenterology, Tohoku University School of Medicine, Aobaku, Sendai, Japan
}

Background \& Aims: Tumor progression is promoted by the ability of tumor cells to resist adverse environmental conditions such as hypoxia. We have shown that translational dysregulation contributes to transformed cell growth in malignant cholangiocytes. Translational regulation of gene expression can contribute to an immediate and rapid response to environmental changes such as hypoxia. Thus, our aims were to assess translational mechanisms involved in cell survival during hypoxia and to identify specific translationally regulated proteins involved in the cellular response to hypoxia. Methods: Cell viability and apoptosis in response to hypoxia were assessed in human cholangiocarcinoma cells. Translational processes were deregulated by cycloheximide or rapamycin or by targeted deletion of eukaryotic initiation factor (elF)-4E, a rate-limiting translational initiation factor using small interfering RNA (siRNA). A protein antibody microarray was used to screen for elF-4Edependent proteins expressed during hypoxia. Expression of the X-linked inhibitor of apoptosis (XIAP) was decreased using siRNA. Results: Malignant cholangiocytes are resistant to hypoxia-induced apoptosis. Furthermore, cell survival during hypoxia required protein translation. elF-4E was over expressed in malignant cholangiocytes. Reduction in elF-4E expression by siRNA decreased tumor cell resistance to hypoxia, increased caspase- 3 activation and apoptosis, and decreased cell survival compared with controls. XIAP was identified as a translationally regulated protein expressed during hypoxia. Modulation of XIAP expression by siRNA decreases cell death during hypoxia in vitro and in vivo. Conclusions: Human cholangiocarcinoma cells are highly resistant to hypoxia. Translational regulation of survival proteins such as XIAP is a mechanism mediating cholangiocarcinoma survival during hypoxia.

$\mathrm{T}$ umor cells are characterized by aberrant cellular growth responses to environmental changes that normally serve to maintain tissue homeostasis. Tumor progression is promoted by the ability of tumor cells to resist adverse environmental conditions such as hypoxia or nutrient deprivation that cause cell death. Hypoxia commonly occurs in human tumors, and resistance to hypoxia-induced cell death is a characteristic feature of tumor cells. ${ }^{1,2}$ Indeed, the ability to survive under hypoxic conditions and to maintain an adequate vascular supply are critical factors for tumor growth. ${ }^{2}$ Although chronic hypoxia results in stimulation of angiogenesis and neovascularization, this process is often disorganized and may take several days before adequate tumor tissue oxygenation can be restored. ${ }^{3}$ Thus, the ability of tumor cells to survive during transient or acute hypoxia is critical. Hypoxia also has a profound effect on the response to therapy and influences tumor development. ${ }^{1,4,5}$ However, the cellular mechanisms by which tumor cells acquire resistance to hypoxia are poorly understood.

We have recently begun to study the cellular response of biliary tract epithelia to hypoxia. The epithelial lining of the biliary tract is highly sensitive to apoptosis under conditions of reduced oxygenation. ${ }^{6}$ In contrast, malignant biliary epithelial cells (cholangiocytes) are resistant to hypoxia-induced cell death and therefore suitable for the study of intracellular survival mechanisms during hypoxia. Biliary tract malignancies (cholangiocarcinomas) are typically hypovascular tumors that are associated with a poor prognosis and are highly refractory to conventional therapies. ${ }^{7,8}$ Cell survival during hypoxia may involve activation of cell survival pathways and/or aberrant expression of endogenous apoptosis inhibitory proteins. ${ }^{9-11}$ Targeted interventions based on an understanding of the mechanisms of response to hypoxia may improve therapeutic approaches for cholangiocarcinoma and other tumors.

We have recently shown that translational dysregulation contributes to transformed cell growth in malignant

\footnotetext{
Abbreviations used in this paper: AMC, 7-amino-4-methylcoumarin; eIF, eukaryotic initiation factor; IRES, internal ribosome entry sequence; MAPK, mitogen-activated protein kinase; siRNA, small interfering double-stranded RNA; XIAP, X-linked inhibitor of apoptosis.

(C) 2004 by the American Gastroenterological Association 0016-5085/04/ $\$ 30.00$ doi:10.1053/j.gastro.2004.09.002
} 
cholangiocytes. ${ }^{12}$ Translation is the final step in a series of several processes involved in gene expression. ${ }^{13,14}$ Thus, regulation of gene expression at the translational level allows for an immediate and rapid response to environmental changes such as hypoxia. ${ }^{15}$ Survival of malignant cholangiocytes during hypoxia can be mediated by a rapamycin sensitive pathway. ${ }^{16}$ Rapamycin is a selective inhibitor of the mammalian target of rapamycin protein that regulates translation by phosphorylation and activation of several translational regulatory proteins such as the eukaryotic initiation factor (eIF)-4E-binding protein 1 and p70 S6 kinase. ${ }^{17}$ These observations suggest that dysregulation of translationally regulated genes may contribute to malignant cholangiocyte survival during hypoxia. Therefore, our aims were to assess the role of translational mechanisms in cell survival during hypoxia and to identify specific translationally regulated proteins involved in the cellular response to hypoxia. Our studies identify a critical role for translational regulation of an endogenous antiapoptotic protein, the X-linked inhibitor of apoptosis protein (XIAP), as a mechanism mediating survival of malignant cholangiocytes during hypoxia.

\section{Materials and Methods}

\section{Materials}

Fetal bovine serum and Bradford reagent were obtained from Sigma Chemical Co. (St. Louis, MO). CMRL 1066 media, L-glutamine, and antibiotic-antimycotic mix were from Gibco BRL (Grand Island, NY). Monoclonal antibodies to eIF-4E and phospho-specific antibodies to eIF-4E (Ser 209) were obtained from Cell Signaling (Beverly, MA). Antibodies to XIAP, c-IAP-1, c-IAP-2, tubulin, and $\beta$-actin were obtained from Santa Cruz Biotechnology (Santa Cruz, CA). The protease inhibitor cocktail tablets were obtained from Roche Molecular Biochemicals (Indianapolis, IN). All other reagents were of analytic grade from the usual commercial sources.

\section{Cell Culture}

$\mathrm{KMCH}$ human malignant cholangiocytes were obtained as previously described and cultured in Dulbecco's modified Eagle medium with $10 \%$ fetal bovine serum. ${ }^{18}$ $\mathrm{KMp} 38$ dn cells were generated from $\mathrm{KMCH}$ cells stably transfected with pRc/RSV-Flag MKK3 (Ala) encoding a dominant interfering upstream activator of p38 mitogen-activated protein kinase (MAPK) with double-point mutations in Ser 189 and Thr 193 replaced by Ala. ${ }^{12}$ H69 cells, immortalized human nonmalignant cholangiocytes, were obtained and cultured as previously described. ${ }^{19} \mathrm{Mz}$-ChA-1 cells derived from metastatic gall bladder cancer (kindly provided by Dr. J.G. Fitz, University of Colorado, Denver, CO) and TFK-1 cells derived from extrahepatic cholangiocarcinoma were cultured in CMRL 1066 media with 10\% fetal bovine serum, 1\%
L-glutamine, and $1 \%$ antimycotic antibiotic mix. Cells were cultured in $35-\mathrm{mm}$ or 96 -well culture plates at $37^{\circ} \mathrm{C}$ in a hypoxia chamber (Billups-Rothenburg, Del Mar, CA) left open in a humidified incubator in $21 \% \mathrm{O}_{2}, 5 \% \mathrm{CO}_{2}$, balance $\mathrm{N}_{2}$, (for normoxia studies) or gassed with a preanalyzed gas mixture containing 5\% $\mathrm{CO}_{2} / 95 \% \mathrm{~N}_{2}$ and sealed (for hypoxia studies). ${ }^{16}$ The partial pressure of oxygen in the culture media under these conditions measured with a gas analyzer (278 System; Ciba-Corning, Medfield, MA) was 140 and $40 \mathrm{~mm}$ $\mathrm{Hg}$, respectively, at 24 hours, and there was no significant change in $\mathrm{pH}$.

\section{Viability Assay}

Cells were seeded into 96-well plates $(10,000$ cells/ well) and incubated in a final volume of $200 \mu \mathrm{L}$ medium. Cell viability was assessed using a commercially available tetrazolium bioreduction assay for viable cells (CellTiter 96 Aqueous; Promega, Madison, WI)

\section{Apoptosis Assay}

Morphologic changes indicative of cell death by apoptosis were identified and quantitated by fluorescence microscopy and the use of 4,6-diamidino-2-phenylindole dihydrochloride (DAPI) as previously described. ${ }^{18}$ Fluorescence was visualized using an Olympus BX40 upright fluorescence microscope (Olympus America, Inc., Melville, NY). Apoptotic nuclei were identified by condensed chromatin as well as nuclear fragmentation. At least 300 nuclei in 4 high-power fields were counted.

\section{Caspase-3 Assays}

Caspase- 3 activity was quantitated in cytosolic extracts as previously described. ${ }^{20}$ Briefly, cytosolic extracts were obtained by cell lysis in a hypotonic buffer containing protease inhibitors, followed by homogenization. Caspase activity was then determined fluorometrically using DEVD-7-amino-4methylcoumarin (AMC), and quantitated using standard curves generated with AMC. Activation of caspase- 3 in transfected cells was assessed by an immunocytochemical assay. Cells were harvested and permeabilized and fixed using the Cytofix/Cytoperm kit (BD Biosciences, Palo Alto, CA). The cells were stained with anti-caspase- 3 monoclonal antibody (BD Biosciences), washed, and stained with Cy5-anti-IgG (Jackson Immunoresearch Labs). The cells were also stained with the nucleic acid dye SYTO-16 (Molecular Probes, Eugene, OR). Stained cells were suspended in an isobuoyant cell buffer at $2 \times 10^{6}$ cells $/ \mathrm{mL}$. Ten microliters of the cell suspension were applied to sample wells of the cell assay chip and assayed on the Agilent 2100 Bioanalyzer microfluidic system (Agilent, Palo Alto, CA). Five hundred to 1000 cell events were collected per sample. Fluorescence emission from the cells was detected with photodiodes at 510-540 nm and 674-696 $\mathrm{nm}$. Cell events in the SYTO-16-positive population were cross gated onto the caspase- 3 histogram to determine the percentage of cells undergoing apoptosis. 


\section{Immunoblot Analysis}

Confluent cells in culture were washed with $1 \times$ PBS then lyzed with $0.5 \mathrm{~mL}$ lysis buffer containing $62.5 \mathrm{mmol} / \mathrm{L}$ Tris base, 2\% SDS, $10 \%$ glycerol, $0.01 \%$ bromophenol blue, and $50 \mathrm{mmol} / \mathrm{L}$ DTT. Protein samples were separated on $4 \%-12 \%$ gradient polyacrylamide gels (Novex, San Diego, CA) under reducing conditions and electroblotted to positively charged $0.45 \mu \mathrm{mol} / \mathrm{L}$ nitrocellulose membrane (Millipore, Bedford, CA). The membranes were soaked for 5 minutes in transfer buffer (13.4 mmol/L Tris, $\mathrm{pH} \mathrm{8.3,20 \%} \mathrm{methanol,} 108$ $\mathrm{mmol} / \mathrm{L}$ glycine). Blots were preblocked in $20 \mathrm{mmol} / \mathrm{L}$ Tris, $150 \mathrm{mmol} / \mathrm{L} \mathrm{NaCl}, 0.1 \%$ Tween 20 , and $5 \%$ nonfat dry milk for $3-4$ hours or overnight at $4{ }^{\circ} \mathrm{C}$. Membranes were incubated overnight at $4{ }^{\circ} \mathrm{C}$ with the respective primary antibody, used at a 1:1000 dilution. The primary antibodies were diluted in a solution containing $20 \mathrm{mmol} / \mathrm{L}$ Tris, $150 \mathrm{mmol} / \mathrm{L} \mathrm{NaCl}, 0.1 \%$ Tween 20 , and $5 \%$ bovine serum albumin. The membrane was washed 3 times for 10 minutes with $20 \mathrm{mmol} / \mathrm{L}$ Tris, 150 $\mathrm{mmol} / \mathrm{L} \mathrm{NaCl}$, and $0.1 \%$ Tween 20 (TTBS) and then incubated with the secondary antibody, a polyclonal goat antirabbit immunoglobulin-peroxidase conjugate (Zymed, San Francisco, CA), at a 1:2000 dilution for 60 minutes at $4^{\circ} \mathrm{C}$. The secondary antibody was diluted in TTBS buffer. For all immunoblots, membranes were washed 3 times for 10 minutes with TTBS then visualized using an enhanced chemiluminescence kit (LumiGLO, Cell Signaling, Beverly, MA) following the manufacturer's directions. The relative protein expression was determined by densitometry using a CCD camera-based image analyzer (ChemiImager 4000; Alpha Innotech, San Leandro, CA).

\section{Small Interfering RNA Design, Synthesis, and Transfection}

RNA interference for gene silencing was performed in $\mathrm{KMCH}$ cells using small interfering 21-nucleotide dsRNA (siRNA) molecules as previously described. ${ }^{12}$ siRNA were designed and synthesized using the Silencer siRNA construction kit (Ambion, Austin, TX). Transfection efficiency was $35 \%-40 \%$ in $\mathrm{KMCH}$ cells using Trans-IT TKO (Mirus, Madison, WI) as previously described. ${ }^{21}$ Inhibition of target protein expression after transfection of cells was verified by immunoblot analysis. The messenger RNA (mRNA) target sequence of siRNA to eIF-4E was 5'-, AAGGATGGTATTGAGCCTATG, and the mRNA target of the scrambled nucleotide control was 5'-AAGTGCTAGATTGAGTGCTAG. The mRNA target sequence of siRNA to XIAP was 5' AACTTGCTAACTCTCTTGGGG, and the mRNA target of the corresponding control siRNA was 5'-AATGCGTACTTCGCATCGTTG.

\section{Cell Cycle Analysis}

Cell cycle analysis was performed as previously described. ${ }^{22}$ Cells were collected and suspended in $1 \times$ PBS at a concentration of $2 \times 10^{6}$ cells $/ \mathrm{mL}$. The samples were centrifuged at $400 \mathrm{~g}$ for 3 minutes and then resuspended in a propidium iodide solution containing $0.1 \mathrm{~mol} / \mathrm{L}$ propidium iodide, $0.1 \% \mathrm{vol} / \mathrm{vol}$ Triton $\mathrm{X}-100$, and $20 \%$ RNAse A, in $1 \mathrm{X}$ PBS. After incubation on ice in the dark for 2 hours, the samples were analyzed by flow cytometry. Ten thousand events were recorded, and the proportion of cells in various phases of the cell cycle were analyzed using the ModFitLT DNA analysis program (Becton Dickinson, San Jose, CA).

\section{Protein Antibody Microarray}

Cells were transfected with siRNA to eIF-4E or a scrambled nucleotide control as previously described. ${ }^{12}$ After 48 hours, the media was replaced with serum-free media for 6 hours. Cells were then incubated under hypoxic or normoxic conditions. After 24 hours, cellular protein was extracted, and the relative differences in protein expression were assessed using the antibody microarray (BD Biosciences Clontech, Palo Alto, CA) as per the manufacturer's instructions. Image and data acquisition from the antibody microarray slides was performed using an Axon GenePix 4000A laser scanner and the GenePix 4.1 software package (Axon Instruments, Foster City, CA). Internal normalization was performed following the manufacturer's protocol. A greater than 1.5-fold difference in relative protein expression was considered significant.

\section{RT-PCR Analysis}

Total cellular RNA was extracted from cells using the Ultraspec RNA isolation reagent (Biotecx Laboratories, Inc., Houston, TX) and semiquantitative RT-PCR performed as previously described. ${ }^{22}$ In brief, cDNA was prepared from 2 to $10 \mu \mathrm{g}$ total RNA using Moloney murine leukemia virus (MMLV) reverse transcriptase and random oligonucleotide primers. PCR was then performed using a DNA thermal cycler and a reaction mixture containing $2 \mu \mathrm{L}$ cDNA and using the taq PCR core kit (Qiagen Inc., Valencia, CA). The reaction mixture was incubated at $95^{\circ} \mathrm{C}$ for 2 minutes, followed by 37 , 3-step cycles $\left(94^{\circ} \mathrm{C}\right.$ for 1 minute, $62^{\circ} \mathrm{C}$ for 1 minute, and $72^{\circ} \mathrm{C}$ for 1 minute) and a final step at $72^{\circ} \mathrm{C}$ for 10 minutes. For GAPDH, 25 cycles were used. The primers used were as follows: human XIAP 5'-GGCCATCTGAGACACATGCAG-3' (sense) and 5'-GCATTCACTAGATCTGCAACC-3' (antisense); GAPDH 5'-TGCCAGTGAGCTTCC-3' (sense) and $5^{\prime}$-CACCATGGAGAAGGC-3' (antisense). The products were analyzed using the Agilent 2100 Bioanalyzer (Agilent, Palo Alto, CA), and gene expression of XIAP was normalized against GAPDH.

\section{Cholangiocarcinoma Xenograft Model}

Male athymic nu/nu mice, 8 weeks of age, were obtained from Charles River Laboratories (Wilmington, MA) and fed food and water ad libitum. The mice were housed 4 per cage, and fluorescent light was controlled to provide alternate light and dark cycles of 12 hours each. The animals received a subcutaneous injection of Mz-ChA-1 cells $\left(3 \times 10^{6}\right.$ viable cells suspended on $0.5 \mathrm{~mL}$ extracellular matrix gel) on their right flank. After 12 weeks, mice ( $\mathrm{n}=2$ each) were randomly assigned to receive $20 \mu \mathrm{g}$ per gram body weight of siRNA to 
XIAP or scrambled control sequence siRNA in $50 \mu \mathrm{L}$ PBS intratumorally, which was repeated after 3 days. Three days later, $60 \mathrm{mg} / \mathrm{kg}$ Hypoxyprobe-1 (Pimonidazole Hydrochloride; Chemicon, Temecula, CA) was injected intravenously, and tumors were excised for histologic examination after 2 hours. Sections were obtained, and immunohistochemistry was performed using monoclonal antibody to XIAP to identify XIAP expression or using Hypoxyprobe-1 monoclonal antibody to identify pimonidazole adducts as a marker of hypoxia. TUNEL staining was performed in adjacent sections to identify apoptotic cells using a commercially available kit (Wako Chemicals, Tokyo, Japan). The number of TUNEL positive cells were quantitated and expressed as average \pm standard deviation of the number of positive cells in 5 high-power fields. The extent of hypoxia was determined by immunostaining for pimonidazole adducts, ${ }^{23}$ and the amount of chromogen quantitated by digital image analysis using Adobe Photoshop. ${ }^{24}$

\section{Statistical Analysis}

Data are expressed as the mean \pm standard deviation (SD) from at least 3 separate experiments, unless otherwise noted. The differences between groups was analyzed using a double-sided Student $t$ test when only 2 groups were present. Statistical significance was considered as $P<.05$. Statistical analyses were performed with the GB-STAT statistical software program (Dynamic Microsystems Inc., Silver Spring, $\mathrm{MD})$.

\section{Results}

\section{Survival of Malignant Cholangiocytes During Hypoxia Is Translationally Regulated}

Initially, we examined the effect of hypoxia on cell viability in $\mathrm{KMCH}$, TFK-1, and $\mathrm{MzChA}-1$ malignant human cholangiocytes or on H69 nonmalignant human cholangiocytes. Similar to results observed in other tumor cell types, cell viability assessed using a tetrazolium bioreduction assay was not decreased in any of the malignant cells during hypoxia (Figure $1 A$ ). Next, we assessed the effect of hypoxia on apoptosis. All 3 malignant cholangiocytes were resistant to hypoxiainduced apoptosis (Figure $1 B$ ). In contrast, hypoxia decreased cell viability and increased apoptosis in H69 nonmalignant human cholangiocytes. These data suggest that survival of malignant cholangiocytes during hypoxia results from effects on apoptosis. Next, we assessed the effect of inhibitors of RNA and protein synthesis on cell survival during hypoxia (Figure $2 A$ ). Preincubation with the RNA synthesis inhibitor actinomycin D $(10 \mu \mathrm{mol} / \mathrm{L})$ did not alter resistance to hypoxia. However, preincubation with the protein synthesis inhibitors cycloheximide $(10 \mu \mathrm{g} / \mathrm{mL})$ or rapamycin $(2 \mu \mathrm{g} / \mathrm{mL})$ increased cell death during hypoxia. Cell viability was decreased during hy-
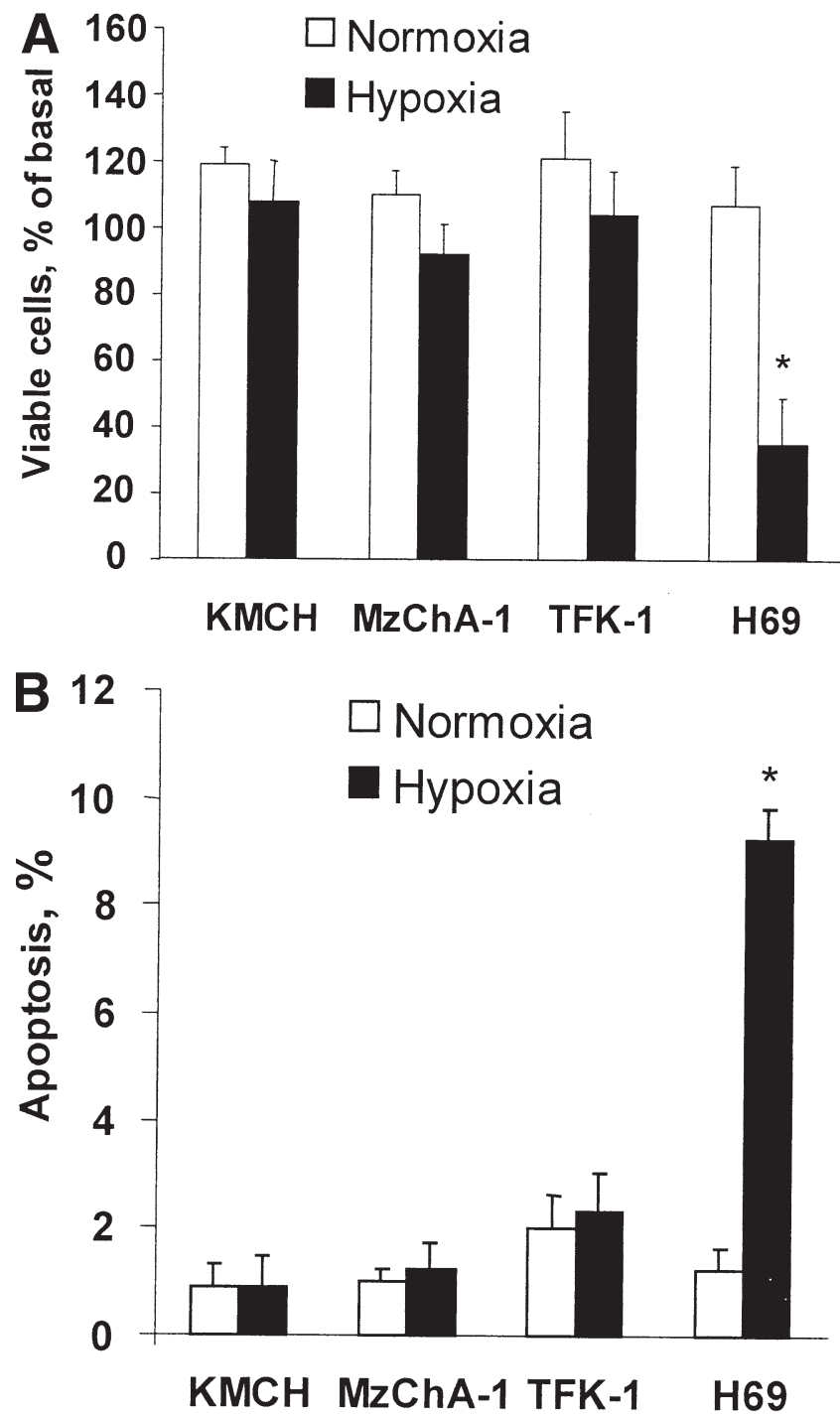

Figure 1. Malignant human cholangiocytes survive during hypoxia. Malignant cholangiocytes (KMCH, Mz-ChA-1 or TFK-1) or nonmalignant cholangiocytes ( $\mathrm{H} 69)$ were cultured under normoxic $\left(21 \% \mathrm{O}_{2}, 5 \% \mathrm{CO}_{2}\right.$, balance $\mathrm{N}_{2}$ ) or hypoxic conditions $\left(95 \% \mathrm{~N}_{2}, 5 \% \mathrm{CO}_{2}\right)$ for 24 hours at $37^{\circ} \mathrm{C}$. (A) Cell viability was assessed using a tetrazolium bioreduction viable cell assay. In contrast to the nonmalignant $\mathrm{H} 69$ cells, the malignant cholangiocyte cell lines were resistant to hypoxia. Results represent the mean \pm standard error of 3 studies. $(B)$ Apoptosis was quantitated by fluorescence microscopy of cells as described in the Materials and Methods section. Malignant cholangiocytes were resistant to hypoxia-induced apoptosis. Results represent the mean \pm standard error of 4 studies. $* P<.05$ compared with normoxia.

poxia to $73 \%$ of normoxia controls with rapamycin and to $76 \%$ of normoxia controls with cycloheximide. Furthermore, activity of caspase- 3 , an effector molecule in cellular apoptosis, was increased in cells preincubated with cycloheximide or rapamycin, but not with actinomycin D (Figure 2B). In combination, these studies indicate that tumor cell resistance to apoptosis and survival during hypoxia involve a translationally regulated response to hypoxia. 
A

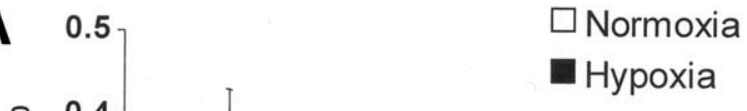

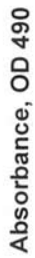

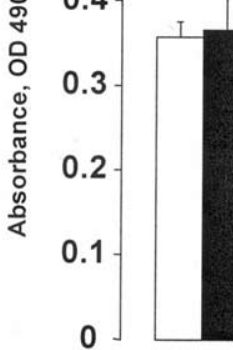

온
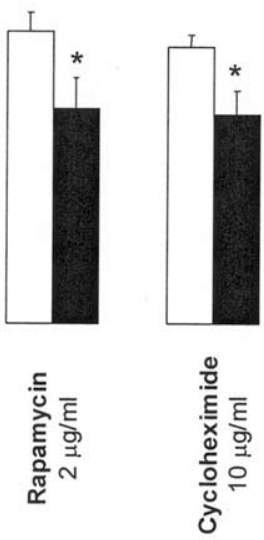
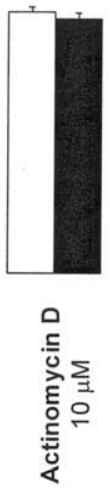

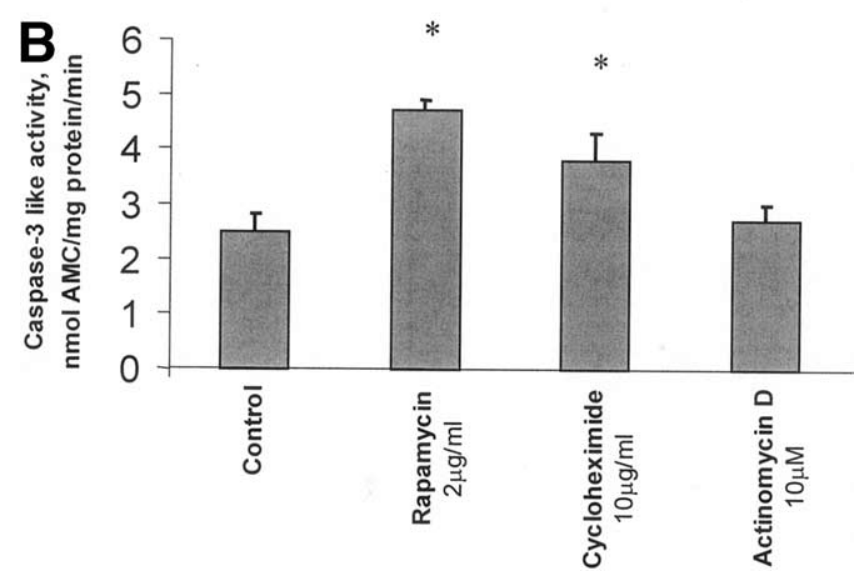

Figure 2. Cell survival during hypoxia requires protein translation. $\mathrm{KMCH}$ cells $\left(10^{5} / \mathrm{mL}\right)$ were plated in 6 -well plates. Cells were pretreated with rapamycin $2 \mu \mathrm{g} / \mathrm{mL}$, cycloheximide $10 \mu \mathrm{g} / \mathrm{mL}$, or actinomycin D, $10 \mu \mathrm{mol} / \mathrm{L}$ for 30 minutes prior to culture under normoxic $\left(21 \% \mathrm{O}_{2}, 5 \% \mathrm{CO}_{2}\right.$, balance $\left.\mathrm{N}_{2}\right)$ or hypoxic conditions $\left(95 \% \mathrm{~N}_{2}, 5 \% \mathrm{CO}_{2}\right)$ at $37^{\circ} \mathrm{C}$. (A) Cell viability was assessed after 24 hours. Results are expressed as absorbance readings at $490 \mathrm{~nm}$ as a direct measurement of metabolically active, viable cells and represent mean \pm standard error of 3 studies. Preincubation with the protein synthesis inhibitors decreased cell viability during hypoxia. $* P<.05$ compared with normoxia controls. (B) Caspase-3-like activity in cells incubated under hypoxic conditions for 24 hours. There was no significant difference in caspase-3-like activity between the groups in cells incubated under normoxic conditions. However, an increase in caspase3-like activity is observed during hypoxia in cells preincubated with protein synthesis inhibitors. $* P<.05$ compared with untreated controls.

\section{Expression of elF-4E Is Increased in Malignant Cholangiocytes}

Having shown a requirement for new protein synthesis for cell survival during hypoxia, we examined the effect of hypoxia on the regulation of initiation of protein translation. The translation factor eIF-4E is rate limiting for the initiation of protein translation and is an important regulator of mRNA translation and protein synthesis. eIF-4E binds to the cap structure at the $5^{\prime}$-end of mRNA of eukaryotic mRNA as a component of the cap-binding complex eIF-4F. The eIF-4F complex mediates the recruitment of ribosomes to mRNA, a ratelimiting step for translation to occur. Indeed, eIF-4E is up-regulated in human cholangiocarcinoma and in the malignant cholangiocyte cell lines used in our study (Figure 3). ${ }^{25}$ Furthermore, eIF-4E expression was not altered, but phosphorylation was increased during hypoxia (data not shown). We have shown that p38 MAPK signaling contributes to translational regulation of growth in malignant cholangiocytes. ${ }^{12}$ Because p38 MAPK-signaling pathways can phosphorylate eIF-4E, we investigated the role of this pathway on eIF-4E phosphorylation during hypoxia. However, eIF-4E phosphorylation during hypoxia was not altered in KM-p38dn cells, which are derived from $\mathrm{KMCH}$ cells and have a functional defect in p38 MAPK activation. Thus, hypoxia increases eIF-4E phosphorylation by a p38 MAPK-independent pathway.

\section{Resistance to Apoptosis During Hypoxia Is elF-4E Dependent}

To assess directly the role of eIF-4E and translationally regulated mechanisms in the cellular response to hypoxia, we used siRNA to functionally decrease eIF-4E expression as described. ${ }^{12}$ siRNA to eIF-4E significantly decreased tumor cell resistance to hypoxia-induced cell death (Figure 4). Furthermore, siRNA to eIF-4E increased caspase- 3 activation and apoptosis during hypoxia compared with scrambled

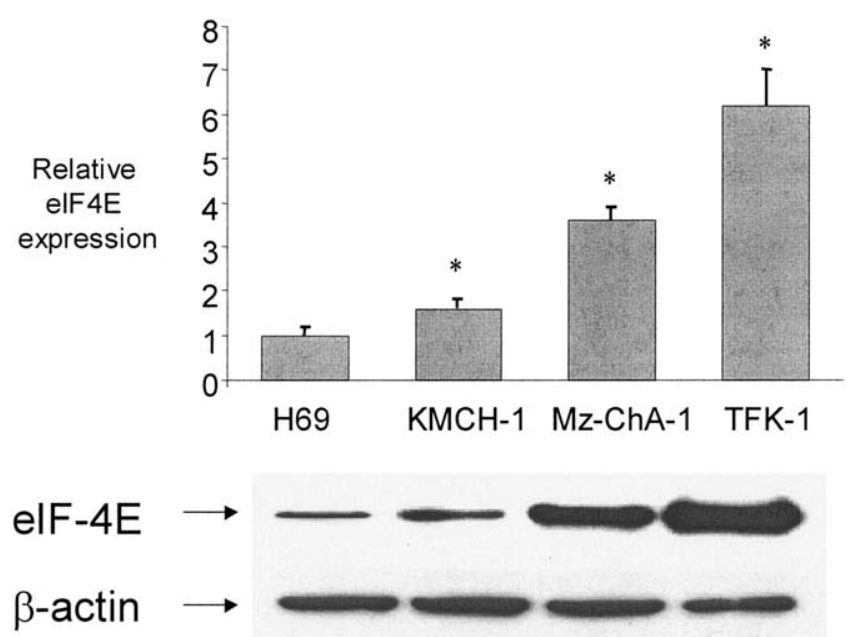

Figure 3. Immunoblot analysis of elF4E expression in human cholangiocytes. elF-4E expression was assessed in H69; nonmalignant human cholangiocytes; or in $\mathrm{KMCH}, \mathrm{Mz}-\mathrm{ChA}-1$, or TFK-1 malignant human cholangiocytes. A representative immunoblot is illustrated below, and the mean \pm standard deviation of expression relative to H69 nonmalignant cholangiocytes of 3 separate experiments is presented in the graph above. $* P<.05$ compared with $\mathrm{H} 69$ cells. 
Normoxia

\section{Hypoxia}
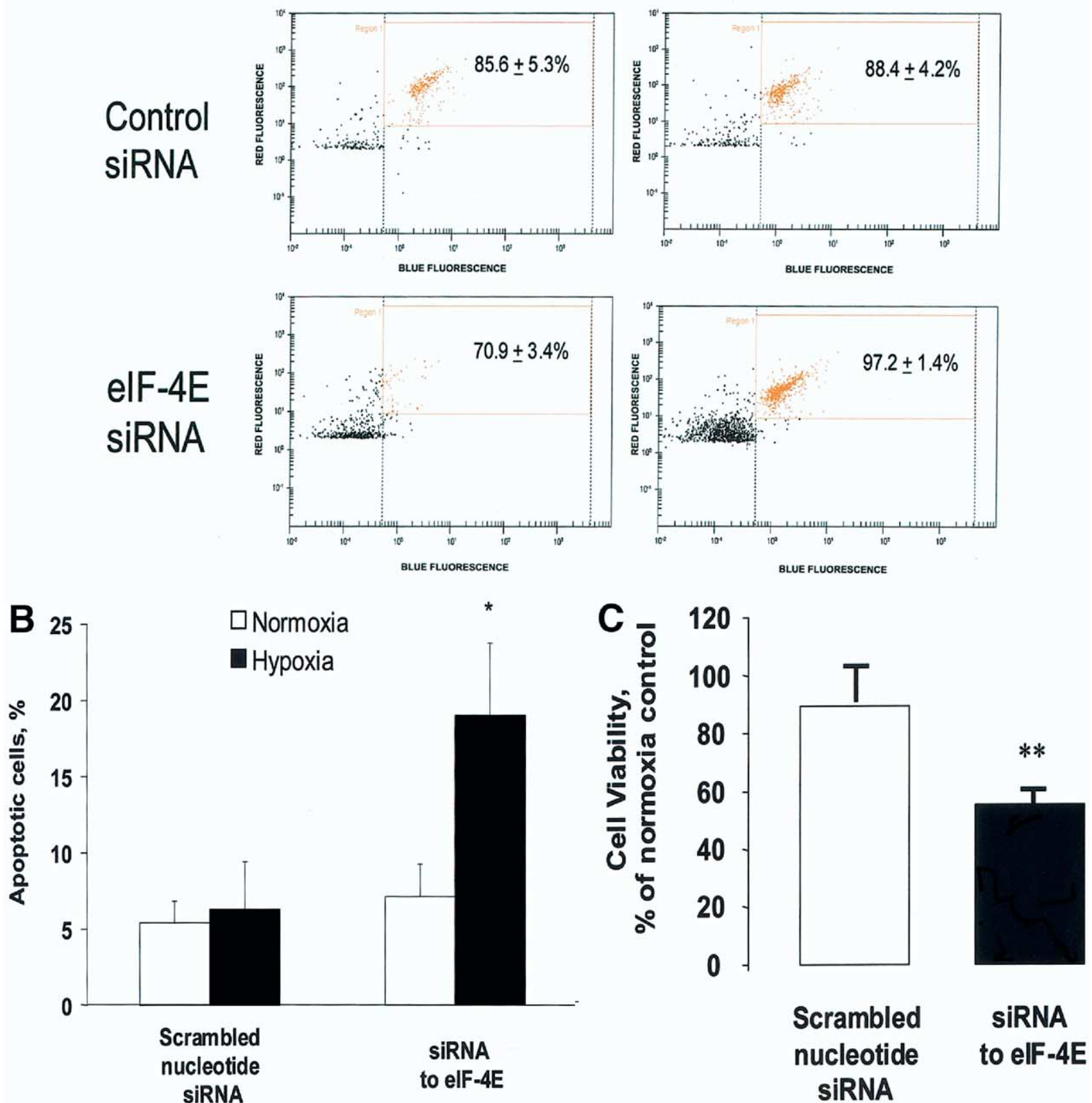

Figure 4. Caspase-3 activation and cell survival during hypoxia are elF-4E dependent. KMCH cells were transiently transfected with siRNA to elF-4E or a scrambled nucleotide siRNA for 48 hours. The media was changed, and cells were cultured under hypoxic or normoxic conditions for 24 hours. (A) Cells were fixed and permeabilized and then stained with antiactive caspase 3 monoclonal antibody/Cy5anti-lgG and with the nucleic acid dye SYTO-16. Cells were assayed for activated caspase-3 using the Agilent 2100 Bioanalyzer microfluidic system. Cell events in the SYTO-16-positive population (blue channel) were cross gated onto the caspase-3 histogram (red channel) to determine the percentage of apoptotic cells. Cells transfected with siRNA to elF-4E showed increased caspase-3 activation during hypoxia compared with controls. The mean and standard deviation of 4 separate determinations are shown. (B) Apoptosis was assessed by fluorescence microscopy after staining cells with DAPI. The number of cells showing morphologic features of apoptosis was quantitated in at least 300 cells in 4 or more high-power fields. The results represent mean \pm standard error of 4 studies. $* P<.05$ compared with normoxia control. $(C)$ Cell viability was assessed using a viable cell assay. Incubation with siRNA to elF-4E decreased viability during hypoxia compared with controls. Results are expressed as cell viability during hypoxia as a percentage of viability in normoxia controls and represent mean \pm standard error of 3 studies. $* * P<.05$ compared with scrambled nucleotide siRNA control. 
Table 1. Hypoxia Delays Cell Cycle Progression

\begin{tabular}{lllrr}
\hline & & GO-G1 & S & G2-M \\
\hline Untransfected cells & Normoxia & $72.2 \pm 5.6$ & $17.6 \pm 2.9$ & $10.2 \pm 3.2$ \\
& Hypoxia & $86.1 \pm 0.7$ & $11.5 \pm 0.5$ & $2.4 \pm 0.2$ \\
Control siRNA & Normoxia & $74.4 \pm 1.3$ & $19.0 \pm 1.2$ & $6.7 \pm 0.6$ \\
SiRNA to elF-4E & Hypoxia & $80.4 \pm 0.3$ & $18.4 \pm 1.0$ & $1.2 \pm 1.1$ \\
& Normoxia & $73.1 \pm 0.5$ & $20.4 \pm 0.8$ & $6.5 \pm 0.4$ \\
\hline
\end{tabular}

NOTE. Cell cycle progression following serum stimulation was assessed in untransfected KMCH cells or cells transfected with either siRNA to elF-4E or scrambled nucleotide siRNA control. Cells were incubated under normoxic or hypoxic conditions for 24 hours, and the cell cycle profile was determined by flow cytometry after staining with propidium iodide. Cells in G0-G1, S, and G2-M phases of the cell cycle are expressed as percentages of the total cell population. The figures represent average \pm standard deviation from 3 experiments.

nucleotide controls. Thus, resistance to apoptosis in malignant human cholangiocytes is mediated by eIF$4 \mathrm{E}-$ dependent translation.

\section{Cell Cycle Progression During Hypoxia}

Because growth arrest is an important response to hypoxia, we next determined whether translational mechanisms were involved in mitogenic regulation of cell cycle progression during hypoxia. Hypoxia decreased cell cycle progression in $\mathrm{KMCH}$ cells with a failure to progress to S-phase and an increase in the proportion of cells in the G0-G1 phase. Serum-stimulation of KMCH cells increased the percentage of cells in S-phase from $10.7 \% \pm 0.8 \%$ under basal, serum-starved conditions to $17.2 \% \pm 0.5 \%$ after 24 hours. However, siRNA to eIF-4E did not significantly alter cell cycle progression following serum stimulation under normoxic or hypoxic conditions (Table 1). Thus, delayed cell cycle progression during hypoxia is not modulated by the translation initiation factor eIF-4E.

\section{Hypoxia Increases XIAP Expression}

To assess potential eIF-4E-regulated proteins involved in protection from cell death during hypoxia, we used a protein antibody microarray to screen for protein expression during hypoxia in $\mathrm{KMCH}$ cells transfected with siRNA to eIF-4E or a scrambled nucleotide siRNA. The protein antibody screen identified an increase in protein expression of XIAP during hypoxia. XIAP is an endogenous antiapoptotic protein and a member of an evolutionarily conserved family of genes involved in the regulation of apoptosis that have been linked to cancer. ${ }^{26}$ The results of the antibody screen were confirmed by immunoblotting in cytoplasmic extracts from cells incubated under hypoxic or normoxic conditions. XIAP expression is increased in
A

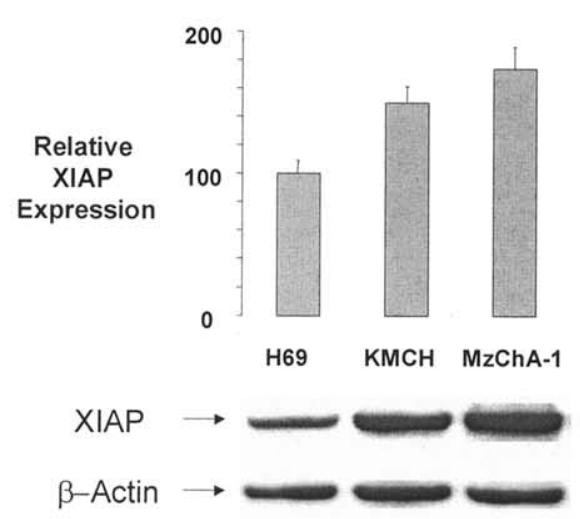

B

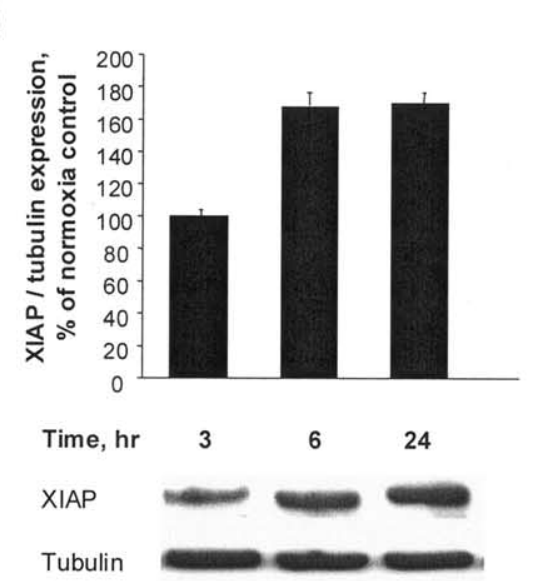

C

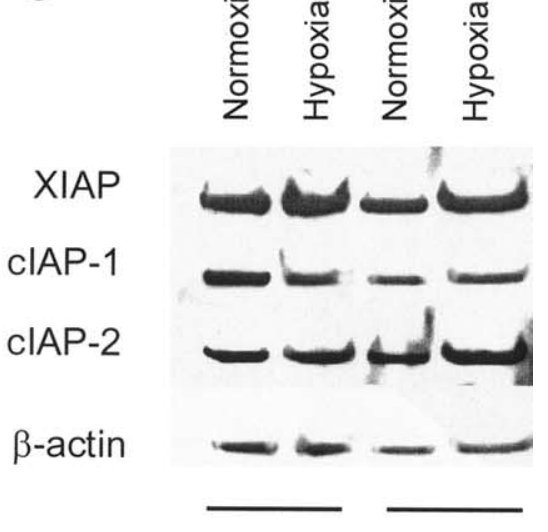

MzChA1 KMCH

Figure 5. XIAP protein expression is increased during hypoxia. (A) XIAP expression was assessed in $\mathrm{H} 69$ (nonmalignant) and $\mathrm{KMCH}$ and Mz-ChA-1 (malignant) cholangiocytes. A representative immunoblot is illustrated, and the graph represents the mean \pm standard deviation of relative expression from 3 separate determinations. (B) KMCH cells were exposed to varying periods of hypoxia. At the indicated times, samples were obtained for immunoblot analysis for XIAP. (C) The expression of inhibitor of apoptosis proteins XIAP, cIAP-1, and clAP-2 was assessed in KMCH and Mz-ChA-1 human malignant cholangiocytes incubated under normoxic $(\mathrm{N})$ and hypoxic conditions $(\mathrm{H})$ for 24 hours. $\mathrm{A}$ representative immunoblot of 3 separate studies is shown. Hypoxia increased the expression of XIAP but did not alter expression of either clAP-1 or clAP2. 
A

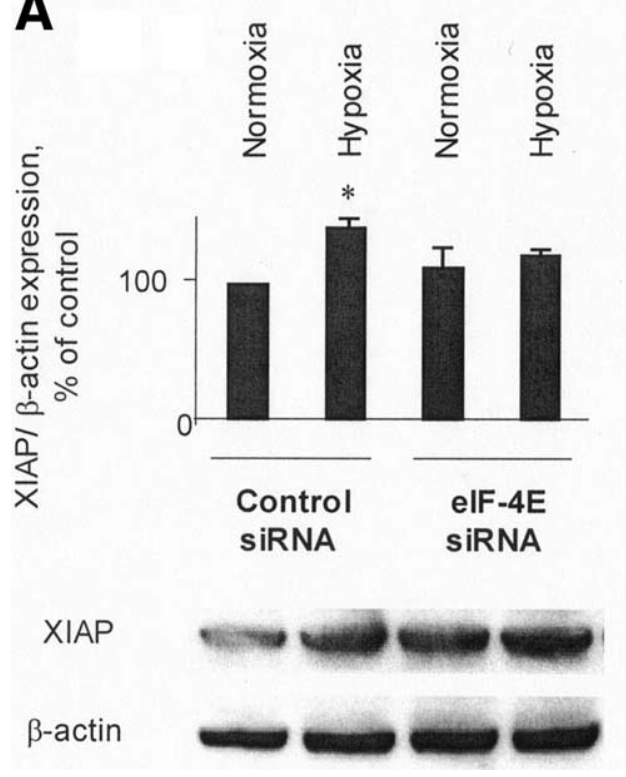

B

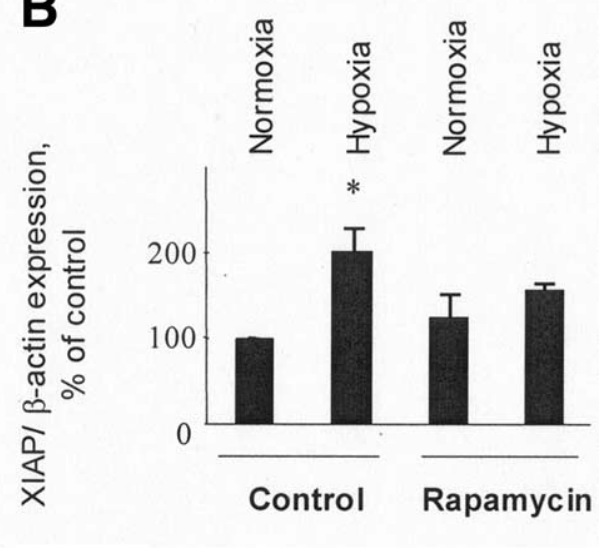

XIAP

$\beta$-actin

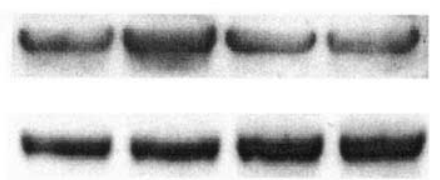

Figure 6. Translational dysregulation decreases XIAP expression during hypoxia. The expression of XIAP was assessed in KMCH malignant cholangiocytes incubated under normoxic or hypoxic conditions for 24 hours. (A) XIAP expression is inhibited in cells transfected with siRNA to elF-4E compared with controls transfected with a scrambled nucleotide control. (B) Pretreatment with the translational inhibitor rapamycin inhibited the increase in XIAP compared with diluent (DMSO) controls. A representative immunoblot is shown, and quantitative data from 3 separate studies are depicted in the graph. $* P<.01$ compared with control.

malignant human cholangiocytes (Figure $5 A$ ). During hypoxia, an increase in XIAP expression occurred by 6 hours (Figure $5 B$ ). XIAP protein expression was increased by $232 \% \pm 27 \%$ of normoxia controls in $\mathrm{KMCH}$ cells and to $192 \% \pm 33 \%$ of normoxia controls in Mz-ChA-1 cells after 24 hours of hypoxia. Protein expression of other apoptosis inhibitor proteins cIAP-1 or cIAP-2 was not increased by hypoxia in either $\mathrm{KMCH}$ or $\mathrm{Mz}-\mathrm{ChA}-1$ malignant human cholangiocytes (Figure 5C). We also assessed XIAP mRNA expression by RT-PCR. In contrast to the increased XIAP protein expression, XIAP mRNA expression was not significantly altered during hypoxia and was $114 \% \pm 7 \%$ and $74 \% \pm 4 \%$ of normoxia controls after 6 and 24 hours of hypoxia, respectively. Furthermore, XIAP expression during hypoxia was decreased in cells transfected with siRNA to eIF-4E or in cells preincubated with rapamycin compared with the relevant controls (Figure 6). XIAP expression can be translationally regulated, and our studies collectively demonstrate translational regulation of XIAP during hypoxia.

\section{XIAP Mediates Cell Survival During Hypoxia}

To assess the role of XIAP in mediating resistance to hypoxia, we used siRNA to decrease cellular XIAP expression. siRNA to XIAP decreased cell viability dur- ing hypoxia compared with a scrambled nucleotide control (Figure 7). XIAP has been previously shown to mediate resistance to radiation induced cell death. ${ }^{27}$ The reduction in cell viability was similar to that observed during incubation with the translational inhibitors cycloheximide or rapamycin but lower that that observed with siRNA to eIF-4E. It is unlikely that XIAP is the only protective factor, given that its inhibition leads to a rather modest sensitization to death, and these data support the presence of additional eIF-4E-dependent factors that are involved in mediating resistance to hypoxia.

The effect of XIAP on tumor cell survival during hypoxia in vivo was assessed in a tumor cell xenograft model in nude mice. Tumors were excised for histologic examination of XIAP, apoptosis, and tissue hypoxia after intratumoral injections of siRNA to XIAP or scrambled nucleotide control siRNA. Illustrative sections are shown in Figure 8. In regions at which the extent of tissue hypoxia was similar, as evidenced by quantitative pimonidazole adduct binding, there was an increase in the number of TUNEL-positive apoptotic cells and a decrease in XIAP expression in tumors that had received siRNA to XIAP compared with those receiving control siRNA (Figure 8). In combination, these results confirm a role for XIAP as an effector of cellular resistance to hypoxia in malignant cholangiocytes in vivo and in vitro. 


\section{Discussion}

Hypoxia is a critical stress for mammalian cells that can result in cell death. Hypoxia commonly occurs in solid human tumors and is associated with a poor prognosis. Indeed, the ability of tumor cells to adapt to the hypoxic microenvironment is a critical stage in malignant progression. Tumor cell adaptations to hypoxia include alterations in cellular metabolism, neovascularization, and ability for the cells to survive in hypoxia. Thus, resistance to hypoxia-induced cell death is a characteristic feature of tumor cells. Understanding tumor cell responses to hypoxia is therefore highly germane to cancer biology. In these studies, we have identified a cellular mechanism contributing to the survival of human malignant cholangiocytes to hypoxia. The principal

\section{Control XIAP SiRNA SiRNA}

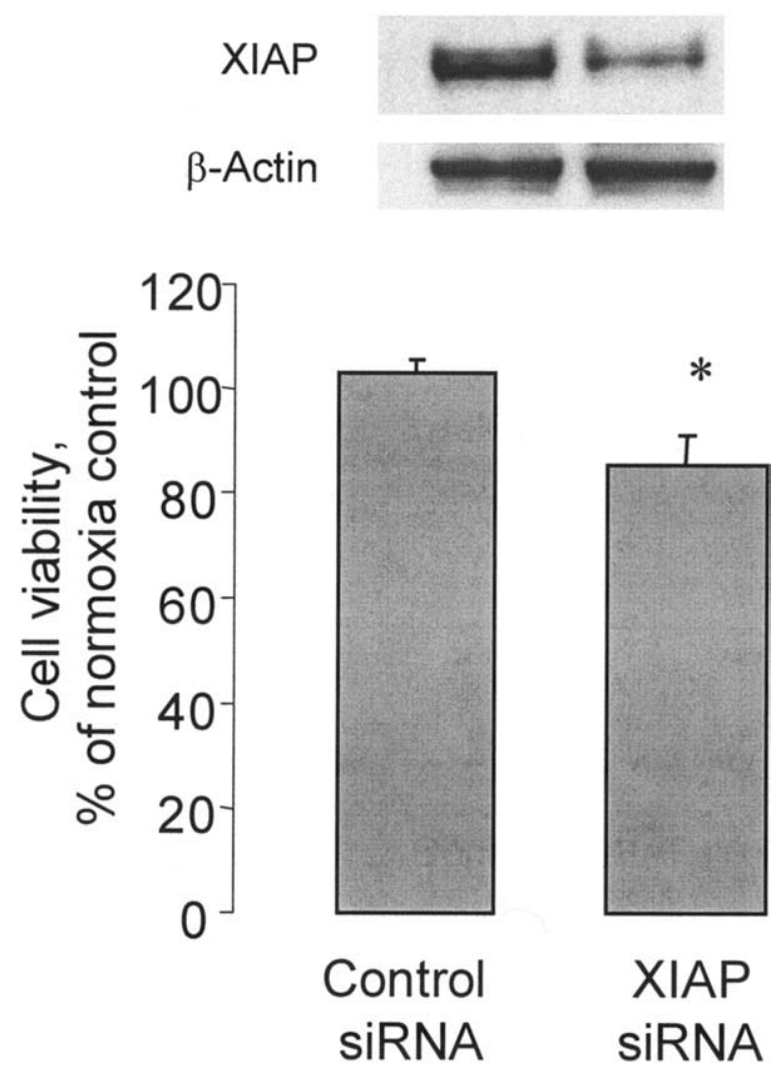

Figure 7. siRNA to XIAP increases cell death during hypoxia. $\mathrm{KMCH}$ cells were transfected with siRNA to XIAP or a scrambled nucleotide (control) siRNA for 48 hours. XIAP expression following transfection was assessed by immunoblot analysis. The media was changed, and cells were then cultured under hypoxic or normoxic conditions for 24 hours. Cell viability was then assessed using a viable cell assay. Incubation with siRNA to XIAP decreased viability during hypoxia compared with controls. Results represent mean \pm standard error of 3 separate studies. ${ }^{*} P<.05$ compared with scrambled nucleotide control.

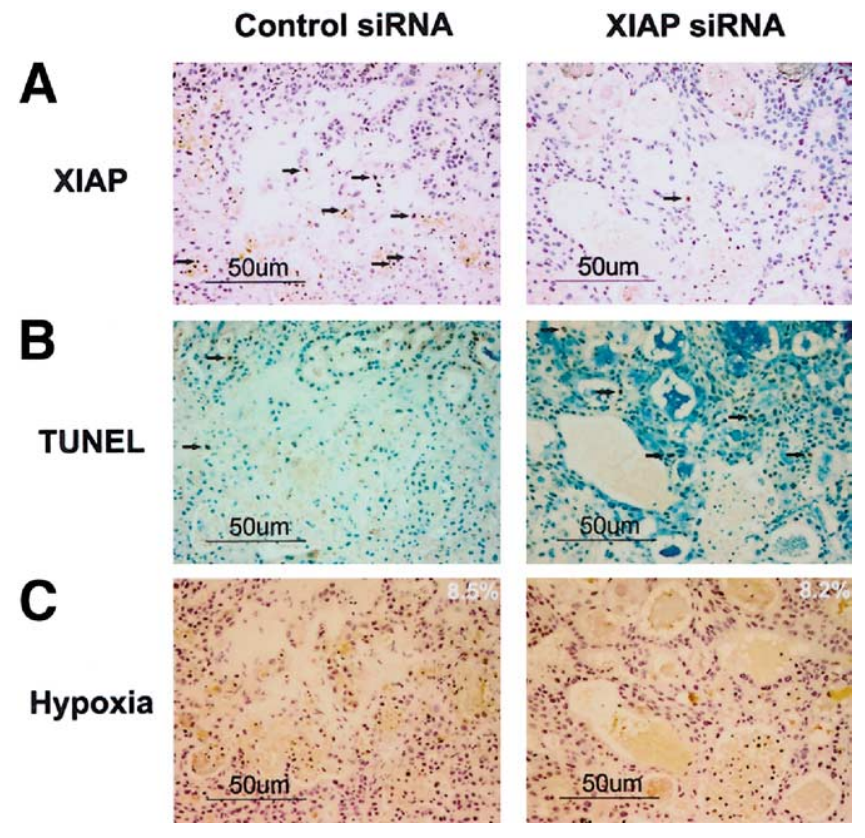

Figure 8. siRNA to XIAP decreases apoptosis in vivo. siRNA to XIAP or a scrambled nucleotide control siRNA were injected into human malignant cholangiocyte xenografts in nude mice as described in the Materials and Methods section, and sections were obtained for analysis of XIAP expression, apoptosis, and hypoxia. Representative adjacent sections are shown following $(A)$ immunohistochemistry for XIAP expression, $(B)$ TUNEL staining for apoptotic cells, and $(C)$ pimonidazole adduct immunostaining for hypoxia. The arrows show XIAP or TUNEL positive staining cells, and the percentage of total area with pimonidazole binding is reported for the lowermost panels. In tumors that had received siRNA to XIAP, there were 20.6 \pm 3.2 TUNELpositive cells per high-power field compared with $7.6 \pm 3.6$ TUNELpositive cells per high-power field in tumors that had received control siRNA ( $\mathrm{n}=5$ fields). Bars represent $50 \mu \mathrm{m}$.

findings are that human malignant cholangiocytes respond to hypoxia by altered translation of the endogenous apoptosis inhibitor XIAP. Dysregulation of protein synthesis and translation by the inhibitors cycloheximide and rapamycin or by targeted deletion of the rate-limiting translational initiation factor eIF-4E decreases XIAP expression and tumor cell resistance to apoptosis during hypoxia. These observations define a mechanism of cell survival involving translational regulation of proteins such as XIAP during otherwise detrimental hypoxic conditions.

The cellular response to hypoxia can involve transcriptional or posttranscriptional mechanisms. Although transcriptional regulation of gene expression during hypoxia has been well characterized, the role of translational mechanisms remains poorly understood. Increasing evidence implicates the translational initiation factor eIF-4E in promoting tumor growth. ${ }^{28-33}$ Expression of eIF-4E is increased several-fold in biliary tract as well as several other malignancies, and modulation of eIF-4E decreases tumor cell growth. ${ }^{25,34}$ Increased eIF-4E ex- 
pression may result in increased efficiency of translation of genes such as XIAP that have a long $5^{\prime}$ untranslated region, which makes cap-dependent translation inefficient. Our observations demonstrating a role for translational regulation of XIAP in the survival of human malignant cholangiocytes during hypoxia are thus consistent with a central role of altered eIF-4E expression in promoting tumor progression through diverse mechanisms.

These studies provide a rationale for additional studies to evaluate the use of translational inhibitors such as rapamycin for the treatment of human cholangiocarcinoma. Rapamycin is a selective inhibitor of FKBP/rapamycin-associated protein (FRAP) or the mammalian target of rapamycin (mTOR), which phosphorylates and activates the translational regulatory protein eIF-4Ebinding protein 1 (4E-BP1) and thereby disrupts its inhibitory interaction with eIF-4E. The critical role of $\mathrm{FRAP} / \mathrm{mTOR}$ in the regulation of translation suggests that specific inhibitors of FRAP/mTOR such as rapamycin, or its analogue CCI-779, which is now in clinical trials, can modulate tumor cell resistance to apoptosis and survival during hypoxia.

Cell stress leading to apoptosis can be associated with inhibition of protein synthesis. Indeed, hypoxia is often associated with a reduction in global protein synthesis in hepatic epithelia. ${ }^{35}$ Thus, the increased translational response during hypoxia is paradoxical. XIAP has a well-characterized internal ribosome entry sequence (IRES) site for translation. ${ }^{36}$ Although IRESmediated translation was initially described for viral RNA as a mechanism for translation of uncapped viral RNA, IRES elements have been described in a few eukaryotic mRNA. IRES-mediated translation occurs during cellular stresses such as exposure to ionizing radiation-induced stress. ${ }^{27,37}$ Although our studies show that the translation of XIAP during hypoxia is dependent on the cap-binding eIF-4E, these observations do not exclude the possibility that IRES-mediated translation also occurs, particularly during acute hypoxia. Further studies are warranted to elucidate the contributions of eIF-4E, and hence cap-dependent translation, and IRES-mediated cap-independent translation during hypoxia.

The present studies provide insight into a potential mechanism by which acquired resistance to hypoxiainduced injury in biliary tract epithelia may promote tumorigenesis. Biliary tract ischemia has been associated with cholangiocyte apoptosis and cell death, suggesting that cholangiocytes are uniquely sensitive to hypoxic injury. Acquired resistance to hypoxia may result from perturbations in critical signaling pathways involved in the cellular response to environmental changes, such as those involving translational factors. Conversely, dysregulation of apoptosis may facilitate the survival of cells with inheritable genetic damage that would have otherwise undergone apoptosis. Furthermore, increased levels of XIAP have been associated with chemoresistance and may contribute to the refractoriness of human cholangiocarcinoma to conventional chemotherapy or to radiation therapy. ${ }^{38-42}$ By activation of cell survival mechanisms, exposure to hypoxia may modulate chemoresistance and tumor aggressiveness and thereby contribute to cholangiocarcinoma progression. Strategies to inhibit tumor cell adaptation to otherwise injurious microenvironmental changes such as hypoxia by manipulating XIAP or eIF-4E expression are attractive novel targets for therapeutic intervention in cholangiocarcinoma.

\section{References}

1. Hockel M, Vaupel P. Biological consequences of tumor hypoxia. Semin Oncol 2001;28:36-41.

2. Hockel M, Vaupel P. Tumor hypoxia: definitions and current clinical, biologic, and molecular aspects. J Natl Cancer Inst 2001; 93:266-276.

3. Bergers G, Benjamin LE. Tumorigenesis and the angiogenic switch. Nat Rev Cancer 2003;3:401-410.

4. Ghafar MA, Anastasiadis AG, Chen MW, Burchardt M, Olsson LE, Xie H, Benson MC, Buttyan R. Acute hypoxia increases the aggressive characteristics and survival properties of prostate cancer cells. Prostate 2003;54:58-67.

5. Subarsky P, Hill RP. The hypoxic tumour microenvironment and metastatic progression. Clin Exp Metastasis 2003;20:237-250.

6. Batts KP. Ischemic cholangitis. Mayo Clin Proc 1998;73:380385.

7. de Groen PC, Gores GJ, LaRusso NF, Gunderson LL, Nagorney DM. Biliary tract cancers. N Engl J Med 1999;341:1368-1378.

8. Gores GJ. Cholangiocarcinoma: current concepts and insights. Hepatology 2003;37:961-969.

9. Horiuchi A, Imai T, Shimizu M, Oka K, Wang C, Nikaido T, Konishi I. Hypoxia-induced changes in the expression of VEGF, HIF-1 $\alpha$, and cell cycle-related molecules in ovarian cancer cells. Anticancer Res 2002;22:2697-2702.

10. Humar R, Kiefer FN, Berns H, Resink TJ, Battegay EJ. Hypoxia enhances vascular cell proliferation and angiogenesis in vitro via rapamycin (mTOR)-dependent signaling. FASEB J 2002;16:771780.

11. Dong Z, Nishiyama J, Yi X, Venkatachalam MA, Denton M, Gu S, Li S, Qiang M. Gene promoter of apoptosis inhibitory protein IAP2: identification of enhancer elements and activation by severe hypoxia. Biochem J 2002;364:413-421.

12. Yamagiwa $Y$, Marienfeld $C$, Tadlock L, Patel T. Translational regulation by p38 mitogen-activated protein kinase signaling during human cholangiocarcinoma growth. Hepatology 2003;38:158-166.

13. Pain VM. Initiation of protein synthesis in eukaryotic cells. Eur J Biochem 1996;236:747-771.

14. Pestova TV, Kolupaeva VG, Lomakin IB, Pilipenko EV, Shatsky IN, Agol VI, Hellen CU. Molecular mechanisms of translation initiation in eukaryotes. Proc Natl Acad Sci U S A 2001;98: 7029-7036.

15. Kraggerud SM, Sandvik JA, Pettersen EO. Regulation of protein synthesis in human cells exposed to extreme hypoxia. Anticancer Res 1995;15:683-686. 
16. Abraham RT. MTOR as a positive regulator of tumor cell responses to hypoxia. Curr Top Microbiol Immunol 2004;279:299319.

17. Gingras AC, Raught B, Sonenberg N. Control of translation by the target of rapamycin proteins. Prog Mol Subcell Biol 2001;27: 143-174.

18. Park J, Tadlock L, Gores GJ, Patel T. Inhibition of interleukin 6-mediated mitogen-activated protein kinase activation attenuates growth of a cholangiocarcinoma cell line. Hepatology 1999; 30:1128-1133.

19. Park J, Gores GJ, Patel T. Lipopolysaccharide induces cholangiocyte proliferation via an interleukin-6-mediated activation of $\mathrm{p} 44 /$ p42 mitogen-activated protein kinase. Hepatology 1999;29:10371043.

20. Tadlock L, Yamagiwa Y, Marienfeld C, Patel T. Double-stranded RNA activates a p38 MAPK-dependent cell survival program in biliary epithelia. Am J Physiol Gastrointest Liver Physiol 2003; 284:G924-G932.

21. Yamagiwa Y, Marienfeld C, Meng F, Holcik M, Patel T. Translational regulation of $\mathrm{x}$-linked inhibitor of apoptosis protein by interleukin-6: a novel mechanism of tumor cell survival. Cancer Res 2004;64:1293-1298.

22. Tadlock L, Patel T. Involvement of p38 mitogen-activated protein kinase signaling in transformed growth of a cholangiocarcinoma cell line. Hepatology 2001;33:43-51.

23. Arteel GE, Thurman RG, Yates JM, Raleigh JA. Evidence that hypoxia markers detect oxygen gradients in liver: pimonidazole and retrograde perfusion of rat liver. Br J Cancer 1995;72:889895.

24. Matkowskyj KA, Cox R, Jensen RT, Benya RV. Quantitative immunohistochemistry by measuring cumulative signal strength accurately measures receptor number. J Histochem Cytochem 2003; 51:205-214.

25. Hansel DE, Rahman A, Hidalgo M, Thuluvath PJ, Lillemoe KD, Shulick R, Ku JL, Park JG, Miyazaki K, Ashfaq R, Wistuba II, Varma R, Hawthorne L, Geradts J, Argani P, Maitra A. Identification of novel cellular targets in biliary tract cancers using global gene expression technology. Am J Pathol 2003;163:217-229.

26. LaCasse EC, Baird S, Korneluk RG, MacKenzie AE. The inhibitors of apoptosis (IAPs) and their emerging role in cancer. Oncogene 1998;17:3247-3259.

27. Holcik M, Yeh C, Korneluk RG, Chow T. Translational upregulation of X-linked inhibitor of apoptosis (XIAP) increases resistance to radiation induced cell death. Oncogene 2000;19:4174-4177.

28. Berkel HJ, Turbat-Herrera EA, Shi R, de Benedetti A. Expression of the translation initiation factor elF4E in the polyp- cancer sequence in the colon. Cancer Epidemiol Biomarkers Prev 2001; 10:663-666.

29. DeFatta RJ, Nathan CA, de Benedetti A. Antisense RNA to elF4E suppresses oncogenic properties of a head and neck squamous cell carcinoma cell line. Laryngoscope 2000;110:928-933.

30. DeFatta RJ, Turbat-Herrera EA, Li BD, Anderson W, de Benedetti A. Elevated expression of elF4E in confined early breast cancer lesions: possible role of hypoxia. Int J Cancer 1999;80:516522.

31. Gingras AC, Raught B, Sonenberg N. elF4 initiation factors: effectors of mRNA recruitment to ribosomes and regulators of translation. Annu Rev Biochem 1999;68:913-963.
32. Graff JR, Zimmer SG. Translational control and metastatic progression: enhanced activity of the mRNA cap-binding protein elF-4E selectively enhances translation of metastasis-related mRNAs. Clin Exp Metastasis 2003;20:265-273.

33. de Benedetti A, Rhoads RE. Overexpression of eukaryotic protein synthesis initiation factor $4 \mathrm{E}$ in HeLa cells results in aberrant growth and morphology. Proc Natl Acad Sci U S A 1990;87: 8212-8216.

34. de Benedetti A, Harris AL. elF4E expression in tumors: its possible role in progression of malignancies. Int J Biochem Cell Biol 1999;31:59-72.

35. Tinton SA, Buc-Calderon PM. Hypoxia increases the association of $4 \mathrm{E}$-binding protein 1 with the initiation factor $4 \mathrm{E}$ in isolated rat hepatocytes. FEBS Lett 1999;446:55-59.

36. Holcik M, Lefebvre C, Yeh C, Chow T, Korneluk RG. A new internal-ribosome-entry-site motif potentiates XIAP-mediated cytoprotection. Nat Cell Biol 1999;1:190-192.

37. Nevins TA, Harder ZM, Korneluk RG, Holcik M. Distinct regulation of internal ribosome entry site-mediated translation following cellular stress is mediated by apoptotic fragments of elF4G translation initiation factor family members elF4GI and p97/ DAP5/NAT1. J Biol Chem 2003;278:3572-3579.

38. Bilim V, Kasahara T, Hara N, Takahashi K, Tomita Y. Role of XIAP in the malignant phenotype of transitional cell cancer (TCC) and therapeutic activity of XIAP antisense oligonucleotides against multidrug-resistant TCC in vitro. Int J Cancer 2003;103:29-37.

39. Sasaki H, Sheng $Y$, Kotsuji F, Tsang BK. Down-regulation of $X$-linked inhibitor of apoptosis protein induces apoptosis in chemoresistant human ovarian cancer cells. Cancer Res 2000;60: 5659-5666.

40. Mansouri A, Zhang Q, Ridgway LD, Tian L, Claret FX. Cisplatin resistance in an ovarian carcinoma is associated with a defect in programmed cell death control through XIAP regulation. Oncol Res 2003;13:399-404.

41. Li J, Feng Q, Kim JM, Schneiderman D, Liston P, Li M, Vanderhyden B, Faught W, Fung MF, Senterman M, Korneluk RG, Tsang BK. Human ovarian cancer and cisplatin resistance: possible role of inhibitor of apoptosis proteins. Endocrinology 2001;142:370 380.

42. Hu Y, Cherton-Horvat G, Dragowska V, Baird S, Korneluk RG, Durkin JP, Mayer LD, LaCasse EC. Antisense oligonucleotides targeting XIAP induce apoptosis and enhance chemotherapeutic activity against human lung cancer cells in vitro and in vivo. Clin Cancer Res 2003;9:2826-2836.

Received August 8, 2003. Accepted August 26, 2004

Address requests for reprints to: Tushar Patel, MD, Division of Gastroenterology, Scott and White Clinic, Texas A\&M University Health Science Center, 2401 South 31st Street, Temple, Texas 76502. e-mail: tpatel@swmail.sw.org; fax: (254) 724-8276.

Supported by the Scott and White Hospital Foundation, grant DK069370 from the National Institutes of Health (to T.P.), and Grantin-Aid for Scientific Research C(16590573) (to Y.U.).

The authors thank the secretarial assistance of Iris Anderson and Cheryle Walters, the technical assistance provided by Robert Jamroz in microarray imaging, and David Clinton Morgan in flow cytometry. 